Research Paper

\title{
Predictors of Left Ventricle Remodeling: Combined Plasma B-type Natriuretic Peptide Decreasing Ratio and Peak Creatine Kinase-MB
}

\author{
Jen-Te Hsu1, Chang-Min Chung1, Chi-Ming Chu², Yu-Shen Lin¹, Kuo-Li Pan¹, Jung-Jung Chang1, Po-Chang
} Wang1, Shih-Tai Chang1, Teng-Yao Yang1, Shih-Jung Jang3, Tsung-Han Yang4, Ju-Feng Hsiao ${ }^{\circledR}$

1. The Department of Cardiology, Chiayi Chang Gung Memorial Hospital, Chang Gung University College of Medicine, Taiwan

2. Section of Health Informatics, Institute of Public Health, National Defense Medical Center and University, Taiwan.

3. The Department of Cardiology, Taipei Tzu Chi General Hospital, Taiwan.

4. Department of Laboratory Medicine, Chang-Gung Medical Foundation.

$\triangle$ Corresponding author: Ju-Feng Hsiao, MD. Department of Cardiology, Chiayi Chang Gung Memorial Hospital, Address: 6, Sec. West Chai-Pu Road, Pu-Tz City, Chai Yi Hsien, Taiwan. Tel: 886-5-3621000 Ext 2854; FAX: 886-5-3623005; E-mail: likeandwind@gmail.com.

(c) Ivyspring International Publisher. This is an open access article distributed under the terms of the Creative Commons Attribution (CC BY-NC) license (https://creativecommons.org/licenses/by-nc/4.0/). See http://ivyspring.com/terms for full terms and conditions.

Received: 2016.08.09; Accepted: 2016.11.24; Published: 2017.01.15

\begin{abstract}
Background: Previous studies reported that patients who had an acute myocardial infarction (AMI) have found that measuring B-type natriuretic peptide (BNP) during the subacute phase of left ventricular (LV) remodeling can predict the possible course of LV remodeling. This study assessed the use of serial BNP serum levels combined with early creatine kinase-MB (CK-MB) to predict the development of significant LV remodeling in AMI patients.

Methods: Nighty-seven patients with new onset AMI were assessed using serial echocardiographic studies and serial measurements of BNP levels, both performed on day-2 (BNP1), day-7 (BNP2), day-90 (BNP3), and day-180 (BNP4) after admission. LV remodeling was defined as $>20 \%$ increase in biplane LV end-diastolic volume on day-180 compared to baseline (day-2).

Results: Patients were divided into LV remodeling [LVR(+)] and non LV remodeling [LVR(-)] groups. No first-week BNP level was found to predict remodeling. However, the two groups had significantly different day-90 BNP level $(208.1 \pm 263.7 \mathrm{pg} / \mathrm{ml}$ vs. $82.4 \pm 153.7 \mathrm{pg} / \mathrm{ml}, \mathrm{P}=0.039)$ and significantly different 3-month BNP decrease ratios $\left(R^{2} \mathrm{NP}_{13}\right)(14.4 \pm 92.2 \%$ vs. $69.4 \pm 25.9 \%, \mathrm{P}<0.001)$. The appropriate cut-off value for $R B \mathrm{NP}_{13}$ was $53.2 \%$ ( $\left.\mathrm{AUC}=0.764, \mathrm{P}<0.001\right)$. Early peak CK-MB (cut-off $48.2 \mathrm{ng} / \mathrm{ml} ; A \cup C=0.672 ; P=0.014)$ was another independent predictor of remodeling. Additionally,

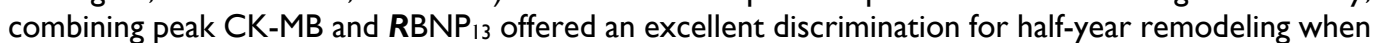
assessed by ROC curve (AUC $=0.818, P<0.001$ ).

Conclusion: $R_{B N P}$ is a significant independent predictor of 6-month LV remodeling. The early peak CK-MB additionally offered an incremental power to the predictions derived from serial BNP examinations.
\end{abstract}

Key words: B-type Natriuretic Peptide (BNP); left ventricular remodeling; acute myocardial infarction (AMI); $\mathrm{BNP}$ decrease ratio; peak creatine kinase-MB.

\section{Introduction}

Left ventricle $(\mathrm{LV})$ remodeling is a complex pathologic process of progressive dilatation, leading to dysfunction and heart failure in patients having had an acute myocardial infarction (AMI). [1] Previous studies have shown B-type natriuretic peptide (BNP) levels can be used for prognostic purposes when measured in the subacute phase of LV remodeling in AMI patients. [2]

This study assessed value of serial measurements of BNP serum levels in predicting LV 
function. To do this, we performed a series of four blood samplings and echocardiographic studies over a six-month period in AMI patients undergoing revascularization.

\section{Methods}

\section{Patients}

The protocol for this study was approved by the ethics committee of the Chiayi Chang Gung Memorial Hospital, and written informed consent was obtained from each patient prior to participation in the study.

This study assessed all patients with new onset AMI admitted to Chiayi Chang Gung Memorial Hospital, Taiwan, between March 2010 and December 2014. We enrolled 110 patients, 97 of whom completed a 6-month echocardiographic follow-up (Fig. 1). Patients with significant mitral regurgitation (MR) secondary to papillary muscle rupture, recent acute coronary syndrome ( $<3$ months), cognitive disorders, or coexisting terminal illness were excluded. Serum concentrations of BNP were measured on day-2 (BNP1), day-7 (BNP2), day-90 (BNP3), and day-180 (BNP4) after admission. Serial echocardiographic studies were also performed four times following the same schedule as BNP sampling.

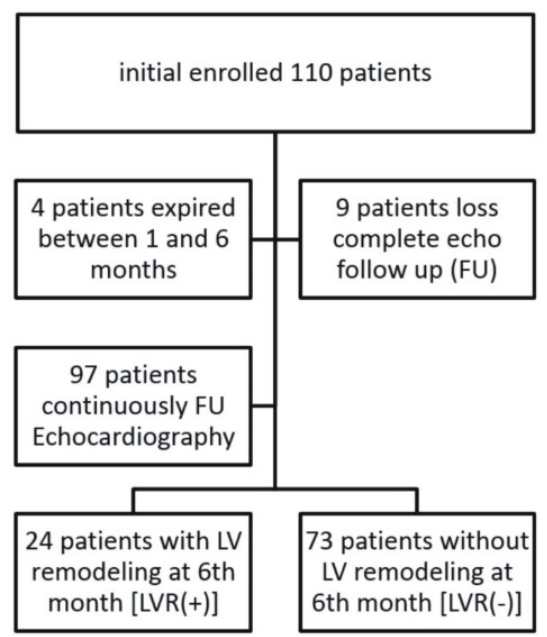

Figure 1. Study flow chart. Ninety-seven patients received 6-month echocardiography and were divided into 2 groups based on presence of LV remodeling (LVR) at 6-month follow-up.

Additional laboratory parameters monitored at first 3 days during hospitalization included lipid profile, liver function panel, serum creatinine level, creatine kinase-MB (CK-MB) and high-sensitivity $\mathrm{C}$-reactive protein (hs-CRP). These parameters were again measured on day-180.

\section{Angioplasty protocol}

Once AMI was diagnosed, percutaneous coronary intervention (PCI) was completed as soon as possible. Patients were classified into two groups: one group with ST-segment elevation myocardial infarction (STEMI, defined as ST elevation $>1 \mathrm{~mm}$ in 2 limb leads or $>2 \mathrm{~mm}$ in leads V1-V6 or new left bundle branch block) and the other group with Non ST-segment elevation myocardial infarction (NSTEMI, defined as no ST-elevation on electrocardiogram (ECG) despite elevated troponin-I $>0.06 \mathrm{ng} / \mathrm{ml}$ ). For STEMI patients, door-to-balloon time was reduced to less than 90 minutes. For NSTEMI patients, PCI was performed as soon as possible and the goal was to reduce door-to-balloon time to less than 48 hours. PCI was considered successful if the residual stenosis was $<30 \%$ and the flow in the involved vessel after the PCI was better than thrombolysis in myocardial infarction (TIMI) grade 2. All patients received dual antiplatelet therapy with a loading dose of aspirin $100 \mathrm{mg}$ and clopidogrel $300 \mathrm{mg}$ and a maintenance dose of aspirin $100 \mathrm{mg}$ and clopidogrel $75 \mathrm{mg}$ per day. When admitted, each patient was prescribed traditional heparin or low molecular weight heparin (enoxaprine) for 2 to 5 days. Heparin treatment was stopped depending upon PCI results and patient symptomatology.

\section{Echocardiographic evaluation}

Echocardiographic examinations (Philip iE33 Ultrasound System) were performed at the same times that blood samples were taken for the measurement of serum BNP. LV systolic function and $\mathrm{LV}$ volume were assessed quantitatively to calculate LV ejection fraction (LVEF) using the modified biplane Simpson's method.

$\mathrm{LV}$ remodeling was defined as $>20 \%$ increase in biplane LV end-diastolic volume (LVEDV) on day-180 compared to baseline day-2. Patients were divided into a $\mathrm{LV}$ remodeling group $[\mathrm{LVR}(+)]$ and a $\mathrm{LV}$ non-remodeling group [LVR (-)] based on the 6-month changes in LVEDV. Both clinical and laboratory data were compared.

\section{Statistical analysis}

All results were expressed as mean \pm SD. Univariate analysis was performed using the Student's t-test. Categorical data were compared against a chi-squared distribution. Spearman's rank correlation coefficients were calculated in order to test the association between variables. Binary logistic regression analysis was used to determine independent variables for $\mathrm{LV}$ remodeling. The predictive value of parameters for detecting LV remodeling was assessed using receiver-operating characteristic (ROC) analysis, identifying the cut-point value that maximized sensitivity and 
specificity. Serial changes in BNP, LVEDV, and LVEF were calculated for the two groups and compared using repeated measures ANOVA. A P value $<0.05$ was considered significant.

\section{Results}

A total of 110 patients were initially enrolled. Four died before the 6-month echocardiography evaluation could be completed. Three of these patients had a cardiac death, one on day-38, another on day-92 and the other day-152. The fourth one had a non-cardiac death on day-79. Nine patients did no complete clinical follow-up. Thus, we were left with 97 patients who completed the half year study and received all clinical, laboratory, and echocardiographic assessments. Based on their 6-month echocardiographic findings, these patients were divided into either a $\mathrm{LV}$ remodeling group $[\operatorname{LVR}(+), \mathrm{n}=24]$ or a LV non-remodeling group [LVR(-), $\mathrm{n}=73$ ] (Fig. 1).

As can be seen in Table 1, a summary of clinical characteristics of the between $\operatorname{LVR}(+)$ and $\operatorname{LVR}(-)$ groups at baseline, there were significant differences in age, door-to-balloon time, symptom-to-balloon time, and STEMI prevalence between the two groups. The LVR(+) group was older than the LVR(-) group $(65.5 \pm 12.0$ vs. $59.8 \pm 12.4, \mathrm{P}=0.05)$. The two groups also had significantly different door-to-balloon (D-to-B) times in hours (7.8 \pm 18.1 vs. $20.4 \pm 30.6, \mathrm{P}=$ 0.018 ) and symptom onset-to-balloon (S-to-B) times in hours $(13.6 \pm 22.3$ vs. $29.1 \pm 35.0, \mathrm{P}=0.015)$.

Table 2 summarizes the coronary artery characteristics and PCI characteristics of the two groups. More bare-metal stents and less drug-eluting stents were deployed among those in the $\operatorname{LVR}(+)$ group than among those in the LVR(-) group ( $\mathrm{P}=$ $0.012)$. Procedural success rate was $100 \%$. Patients in both groups had similar angiographic characteristics, syntax score and stent sizes. There was no significant difference in clinical severity (Killip class) between the two groups.

Laboratory parameters, including BNP, BNP decrease difference $(\triangle \mathrm{BNP}), \mathrm{BNP}$ decrease ratio $(R B N P)$, uric acid, peak creatine kinase-MB fraction (CK-MB) level, and peak troponin I level are summarized in Table 3 . There was no significant difference in baseline first-week BNP measurements (day-2 and day-7 BNP) between the two groups. However, there was a significant difference in day-90 BNP levels between LVR $(+)$ group and LVR(-) group $(208.1 \pm 263.7$ vs. $82.4 \pm 153.7 \mathrm{P}=0.039)$. Another significant difference was found in peak CK-MB $(\mathrm{LVR}(+)$ group $158.8 \pm 156.0$ vs. LVR(-) group $75.8 \pm$ 98.6, $\mathrm{P}=0.023)$.
Table 1. Baseline characteristics in LV remodeling and non LV remodeling groups.

\begin{tabular}{|c|c|c|c|}
\hline Variables & $\operatorname{LVR}(+), \mathrm{n}=24$ & $\operatorname{LVR}(-), \mathrm{n}=73$ & $\mathrm{P}$ \\
\hline Age & $65.5 \pm 12.0$ & $59.8 \pm 12.4$ & 0.050 \\
\hline Male & $19(79.2 \%)$ & $67(91.8 \%)$ & 0.091 \\
\hline Body surface area $\left(\mathrm{m}^{2}\right)$ & $1.7 \pm 0.2$ & $1.8 \pm 0.2$ & 0.501 \\
\hline Body mass index $\left(\mathrm{kg} / \mathrm{m}^{2}\right)$ & $24.7 \pm 3.9$ & $25.2 \pm 3.7$ & 0.602 \\
\hline $\begin{array}{l}\text { Systolic blood pressure } \\
(\mathrm{mmHg})\end{array}$ & $153.5 \pm 26.8$ & $157.3 \pm 29.9$ & 0.577 \\
\hline Heart rate $(\mathrm{bpm})$ & $74.8 \pm 19.4$ & $73.9 \pm 16.1$ & 0.814 \\
\hline D-to-B (hour) & $7.8 \pm 18.1$ & $20.4 \pm 30.6$ & 0.018 \\
\hline S-to-B (hour) & $13.6 \pm 22.3$ & $29.1 \pm 35.0$ & 0.015 \\
\hline STEMI & $17(70.8 \%)$ & $42(57.5 \%)$ & 0.247 \\
\hline Hypertension & $14(58.3 \%)$ & $43(58.9 \%)$ & 0.961 \\
\hline Diabetes mellitus & $7(29.2 \%)$ & $21(28.8 \%)$ & 0.970 \\
\hline Smoking & $12(50.0 \%)$ & $43(58.9 \%)$ & 0.445 \\
\hline Coronary artery disease history & $0(0 \%)$ & $6(8.2 \%)$ & 0.147 \\
\hline $\begin{array}{l}\text { Chronic obstructive pulmonary } \\
\text { disease }\end{array}$ & $0(0 \%)$ & $3(4.1 \%)$ & 0.313 \\
\hline Peripheral vascular disease & $1(4.2 \%)$ & $1(1.4 \%)$ & 0.403 \\
\hline Old stroke & $0(0 \%)$ & $4(5.5 \%)$ & 0.242 \\
\hline Hyperlipidemia & $8(33.3 \%)$ & $25(34.2 \%)$ & 0.935 \\
\hline Renal insufficiency & $2(8.3 \%)$ & $4(5.5 \%)$ & 0.615 \\
\hline Moderate-to-severe MR & $0(0 \%)$ & $2(2.9 \%)$ & 0.445 \\
\hline \multicolumn{4}{|l|}{ Medication } \\
\hline ACEI or ARB & $9(37.5 \%)$ & $33(45.2 \%)$ & 0.509 \\
\hline Beta-blocker & $15(62.5 \%)$ & $37(50.7 \%)$ & 0.314 \\
\hline Statin & $16(66.7 \%)$ & $52(71.2 \%)$ & 0.672 \\
\hline Diuretic & $0(0 \%)$ & $0(0 \%)$ & NA \\
\hline Aldactone & $0(0 \%)$ & $0(0 \%)$ & NA \\
\hline
\end{tabular}

D-to-B: door-to-balloon time; S-to-B: symptom-onset-to-balloon time; STEMI: ST-segment elevation myocardial infarction; ACEI: angiotensin converting enzyme inhibitor; ARB: angiotensin receptor blocker; MR: mitral regurgitation; NA: non-assessment.

Table 2. Characteristics of coronary artery and percutaneous coronary interventions.

\begin{tabular}{|c|c|c|c|}
\hline Variables & $\operatorname{LVR}(+), \mathrm{n}=24$ & $\operatorname{LVR}(-), \mathrm{n}=73$ & $\mathrm{P}$ \\
\hline Target Vessel & & & 0.296 \\
\hline LM & $0(0 \%)$ & $1(1.4 \%)$ & \\
\hline LAD & $8(33.3 \%)$ & $39(53.4 \%)$ & \\
\hline LCX & $3(12.5 \%)$ & $8(11.0 \%)$ & \\
\hline RCA & $13(54.2 \%)$ & $25(34.2 \%)$ & \\
\hline TV characteristics & & & 0.403 \\
\hline A or B1 & $1(4.2 \%)$ & $1(1.4 \%)$ & \\
\hline B2 or C & $23(95.8 \%)$ & $72(98.6 \%)$ & \\
\hline Coronary artery disease & & & 0.925 \\
\hline 1 vessel disease & $7(29.2 \%)$ & $24(32.9 \%)$ & \\
\hline 2 vessel disease & $11(45.8 \%)$ & $33(45.2 \%)$ & \\
\hline 3 vessel disease & $6(25.0 \%)$ & $16(21.9 \%)$ & \\
\hline Syntax score & $21.7 \pm 11.0$ & $19.5 \pm 10.2$ & 0.357 \\
\hline TV stent type* & & & 0.012 \\
\hline BMS & $22(91.7 \%)$ & $43(58.9 \%)$ & \\
\hline DES & $2(8.3 \%)$ & $29(39.7 \%)$ & \\
\hline TV stent size $(\mathrm{mm})$ & $3.3 \pm 0.5$ & $3.3 \pm 0.6$ & 0.605 \\
\hline TV stent length & $34.0 \pm 13$ & $33.5 \pm 16.1$ & 0.882 \\
\hline Killip Class & & & 0.867 \\
\hline I & $17(70.8 \%)$ & $53(72.6 \%)$ & \\
\hline II & $2(8.3 \%)$ & $7(9.6 \%)$ & \\
\hline III & $1(4.2 \%)$ & $5(6.8 \%)$ & \\
\hline IV & $4(16.7 \%)$ & $8(11.0 \%)$ & \\
\hline
\end{tabular}

LM: left main; LAD: left anterior descending artery; LCX: left circumflex artery; RCA: right coronary artery. TV: target vessel; BMS: bare metal stent; DES: drug-eluting stent

* One patient did not accept stent implantation at LVR(-) group. 
We calculated the $\triangle \mathrm{BNP}$ and $R B N P . \Delta \mathrm{BNP}_{1 \mathrm{f}}$ was defined as baseline BNP1 - follow-up BNP; $R B N P_{1 f}$ (\%) was defined as $100 \times$ (baseline BNP1- follow-up BNP)/baseline BNP. The LVR(+) and LVR(-) groups had significant differences in $R_{B N P} P_{13}(14.4 \pm 92.2$ vs. $69.4 \pm 25.9, \mathrm{P}<0.001)$ and $R \mathrm{RNP}_{14}(18.3 \pm 103.2$ vs. 69.3 $\pm 38.9, \mathrm{P}=0.042)$, but no significant differences in other laboratory parameters, including $\triangle \mathrm{BNP}_{12}$, $\triangle \mathrm{BNP}_{13}, \Delta \mathrm{BNP}_{14}, \mathrm{RBNP}_{12}$, uric acid, troponin-I and hs-CRP.

Table 3. Laboratory data including plasma B-type natriuretic peptide (BNP).

\begin{tabular}{|c|c|c|c|}
\hline & $\operatorname{LVR}(+), \mathrm{n}=24$ & $\operatorname{LVR}(-), \mathrm{n}=73$ & $\mathbf{P}^{*}$ \\
\hline BNP1 (day 2) (pg/ml) & $342.1 \pm 369.5$ & $322.0 \pm 440.2$ & 0.841 \\
\hline BNP2 (day 7) (pg/ml) & $327.8 \pm 256.1$ & $224.7 \pm 305.7$ & 0.148 \\
\hline BNP3 (day 90) (pg/ml) & $208.1 \pm 263.7$ & $82.4 \pm 153.7$ & 0.039 \\
\hline BNP4 (day 180) (pg/ml) & $200.2 \pm 335.1$ & $76.6 \pm 155.3$ & 0.124 \\
\hline$\Delta \mathrm{BNP}_{12}(\mathrm{pg} / \mathrm{ml})$ & $-8.9 \pm 248.0$ & $90.3 \pm 248.7$ & 0.099 \\
\hline $\operatorname{RBNP}_{12}(\%)$ & $-40.5 \pm 109.6$ & $-14.7 \pm 179.5$ & 0.518 \\
\hline$\Delta \mathrm{BNP}_{13}(\mathrm{pg} / \mathrm{ml})$ & $110.8 \pm 324.3$ & $246.7 \pm 376.9$ & 0.125 \\
\hline$R_{B N P}{ }_{13}(\%)$ & $14.4 \pm 92.2$ & $69.4 \pm 25.9$ & $<0.001$ \\
\hline$\Delta \mathrm{BNP}_{14}(\mathrm{pg} / \mathrm{ml})$ & $103.9 \pm 298.3$ & $253.6 \pm 420.4$ & 0.141 \\
\hline$R_{B N P}(\%)$ & $18.3 \pm 103.2$ & $69.3 \pm 38.9$ & 0.042 \\
\hline Uric acid (mg/dl) & $9.2 \pm 15.0$ & $6.0 \pm 1.4$ & 0.296 \\
\hline Peak CK-MB (ng/ml) & $158.8 \pm 156.0$ & $75.8 \pm 98.6$ & 0.023 \\
\hline Peak Troponin I (ng/ml) & $12.7 \pm 24.5$ & $7.1 \pm 19.8$ & 0.258 \\
\hline hs-CRP (mg/L) & $37.5 \pm 44.2$ & $33.3 \pm 36.2$ & 0.662 \\
\hline $\begin{array}{l}\left.\text { hs-CRP (6 } 6^{\text {th }} \text { month }\right) \\
\text { (mg/L) }\end{array}$ & $4.5 \pm 12.3$ & $3.9 \pm 7.0$ & 0.782 \\
\hline
\end{tabular}

$\triangle \mathrm{BNP}_{12}=\mathrm{BNP1}-\mathrm{BNP2}$ : one week BNP decrease difference;

$R \mathrm{BNP}_{12}=(\mathrm{BNP} 1-\mathrm{BNP} 2) \times 100 / \mathrm{BNP} 1(\%)$ : one week BNP decrease ratio

$\triangle \mathrm{BNP}_{13}=\mathrm{BNP1}$-BNP3: 3-month BNP decrease difference;

$R \mathrm{BNP}_{13}=(\mathrm{BNP1}-\mathrm{BNP3}) \times 100 / \mathrm{BNP} 1$ (\%): 3-month BNP decrease ratio

$\triangle \mathrm{BNP}_{14}=\mathrm{BNP1}$-BNP4: 6-month BNP decrease difference;

$R \mathrm{BNP}_{14}=(\mathrm{BNP1}-\mathrm{BNP} 4) \times 100 / \mathrm{BNP} 1(\%)$ : 6-month $\mathrm{BNP}$ decrease ratio

$\mathrm{CK}-\mathrm{MB}$ : creatine kinase-MB; hs-CRP: high-sensitivity C-reactive protein

${ }^{*} \mathrm{P}<0.05$ was considered statistically significant.

In this study which aimed to identify independent predisposing factors that might predict half-year remodeling, we excluded the half-year exam or its related parameters $\Delta \mathrm{BNP}_{14}$ and $\mathrm{RBNP}_{14}$. Prior to multivariate analysis, we studied the correlation between $R_{B N P}$ and other variables so that we could eliminate any strongly correlated factors that might mask predicting power of $R_{B N P_{13}}$. Table 4 shows the correlations between $R_{B N P} 13$ and other BNP variables. $\boldsymbol{R B N P}_{13}$ significantly correlated with BNP3, $\triangle \mathrm{BNP}_{12}, \Delta \mathrm{BNP}_{13}$ and $\mathrm{RBNP}_{12}$. Therefore, it was chosen over $\mathrm{BNP} 1, \mathrm{BNP} 2, \mathrm{BNP} 3$ and $\triangle \mathrm{BNP}_{12}$ to test the ability of $\mathrm{BNP}$ to predict $\mathrm{LV}$ remodeling.

Multivariate analysis was used to evaluate all significantly correlated parameters with a $\mathrm{P}$ value $\leq$ 0.05 considered significant. These parameters included age, D-to-B time, S-to-B time, target vessel

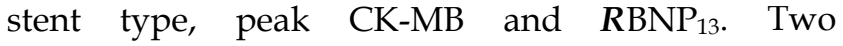
independent predictors of $\mathrm{LV}$ remodeling were found: $R_{B N P} 13$ (odds ratio $=0.972$ [0.954 - 0.990]; $\mathrm{P}<$
0.001) and peak CK-MB (odds ratio = 1.006 [1.001 1.011]; $\mathrm{P}=0.015$ ) (Table 5).

Table 6 showed the serial measurement of echocardiographic parameters including LVEDV, end-systolic volume (ESV) and EF. Because the cut-off value of two LV remodeling groups was defined by the serial change of LVEDV, the multivariate analysis did not include these parameters even with significant differences by independent $\mathrm{T}$ test. The different presentation of echocardiographic measurement was shown both on the table and following figures. Most patients had preserved $\mathrm{EF}$ ( $\mathrm{EF} \geq 40 \%$ ) and only 3 patients had impaired EF $(\mathrm{EF}<40 \%)$ at baseline measurement.

Table 4. Correlation between 3-month plasma B-type natriuretic

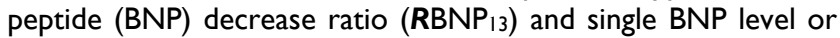
derived parameters (Pearson's correlation coefficient).

\begin{tabular}{|c|c|c|c|c|c|c|}
\hline & BNP1 & BNP2 & BNP3 & $\Delta \mathrm{BNP}_{12}$ & $\mathrm{RBNP}_{12}$ & $\Delta \mathrm{BNP}_{13}$ \\
\hline $\begin{array}{l}\text { Pearson's } \\
\text { correlation } \\
\text { coefficient }(\rho)\end{array}$ & 0.122 & -0.100 & $-0.575^{*}$ & $0.346^{*}$ & $0.206^{*}$ & $0.477^{*}$ \\
\hline $\mathrm{P}$ & 0.228 & 0.325 & $<0.001$ & $<0.001$ & 0.041 & $<0.001$ \\
\hline
\end{tabular}

Table 5. Results of multivariate analysis for half year left ventricle remodeling.

\begin{tabular}{llll}
\hline & OR & $\mathbf{9 0} \% \mathbf{C I}$ & $\mathbf{P}^{*}$ \\
\hline Age & 1.052 & $0.988-1.121$ & 0.112 \\
D-to-B (hour) & 0.969 & $0.912-1.029$ & 0.307 \\
S-to-B (hour) & 1.019 & $0.973-1.066$ & 0.429 \\
TV stent type (DES vs. BMS) & 4.392 & $0.624-30.915$ & 1.000 \\
Peak CK-MB (ng/ml) & 1.006 & $1.001-1.011$ & 0.015 \\
RBNP $_{13}$ & 0.970 & $0.954-0.987$ & $<0.001$
\end{tabular}

OR: odds ratio; CI: confidence interval; S-to-B: symptom-onset-to-balloon time; TV: target vessel; BMS: bare-metal stent; DES: drug-eluting stent; NSTEMI: Non ST-segment elevation myocardial infarction; STEMI: ST-segment elevation myocardial infarction.

${ }^{*} \mathrm{P}<0.05$ was considered significant.

Table 6. Serial measurements of echocardiographic parameters during half year.

\begin{tabular}{lcll}
\hline Variables & $\mathrm{LVR}(+), \mathrm{n}=24$ & $\mathrm{LVR}(-), \mathrm{n}=73$ & $\mathrm{P}$ \\
\hline Ejection fraction $(\%)$ & & & \\
Baseline & $55.7 \pm 10.1$ & $58.1 \pm 9.1$ & 0.279 \\
One week & $55.1 \pm 9.3$ & $60.6 \pm 8.3$ & 0.014 \\
Day 90 & $57.6 \pm 9.9$ & $62.7 \pm 8.2$ & 0.014 \\
Day 180 & $55.9 \pm 8.5$ & $62.8 \pm 8.4$ & 0.002 \\
Left ventricular end-diastolic volume (ml) & & \\
Baseline & $86.1 \pm 21.4$ & $109.5 \pm 28.0$ & $<0.001$ \\
One week & $103.6 \pm 26.1$ & $108.0 \pm 26.8$ & 0.488 \\
Day 90 & $115.0 \pm 30.7$ & $105.5 \pm 27.0$ & 0.153 \\
Day 180 & $126.5 \pm 35.4$ & $103.5 \pm 26.8$ & 0.001 \\
Left ventricular end-systolic volume (ml) & & \\
Baseline & $38.8 \pm 14.7$ & $46.1 \pm 16.3$ & 0.053 \\
One week & $46.9 \pm 19.4$ & $43.0 \pm 16.9$ & 0.352 \\
Day 90 & $49.5 \pm 21.2$ & $40.4 \pm 17.3$ & 0.034 \\
Day 180 & $57.7 \pm 27.5$ & $39.0 \pm 14.5$ & $<0.001$ \\
\hline
\end{tabular}


Repeated-measures analyses of variance (ANOVA) were used to examine patterns of serial plasma B-type natriuretic peptide (BNP) change over the half year (Fig. 2). Pair comparisons revealed significant within-subject effects with regard to BNP 1 vs. BNP3 (P < 0.001) and BNP 1 vs. BNP4 $(\mathrm{P}<0.001)$. In our comparison of BNP changes between the $\operatorname{LVR}(+)$ and LVR(-) groups, we found a significant difference at day $90(\mathrm{P}<0.001)$ and day $180(\mathrm{P}=0.002)$, though we found no interaction and significant between-subjects effects $(P=0.105)$ among the members of these two groups.
Figure 3 shows the correlation between change in 6-month LVEDV dilatation ratio [LV remodeling (\%)] and $R_{B N P} P_{13}$ in all 97 patients (Fig. $3 \mathrm{~A}: \mathrm{P}<0.001$; $\left.\mathrm{R}^{2}=0.150\right)$. The ROC method was used to determine the appropriate cut-off point of $R_{B N P_{13}}(53.2 \%$ ) (Fig. $3 \mathrm{~B}$ : area under the curve $[\mathrm{AUC}]=0.764 ; \mathrm{P}<0.001$ ). Those patients with $R_{B N P} P_{13}$ more than $53.2 \%$ had low risk for developing half-year LV remodeling, otherwise patients with $R_{B N P_{13}}$ less than $53.2 \%$ had potential risk of half-year LV remodeling. The sensitivity of this cut-off value was $81.2 \%$ and the specificity was $60.9 \%$. The positive predictive value was $53.6 \%$ and negative predictive value $87.5 \%$.
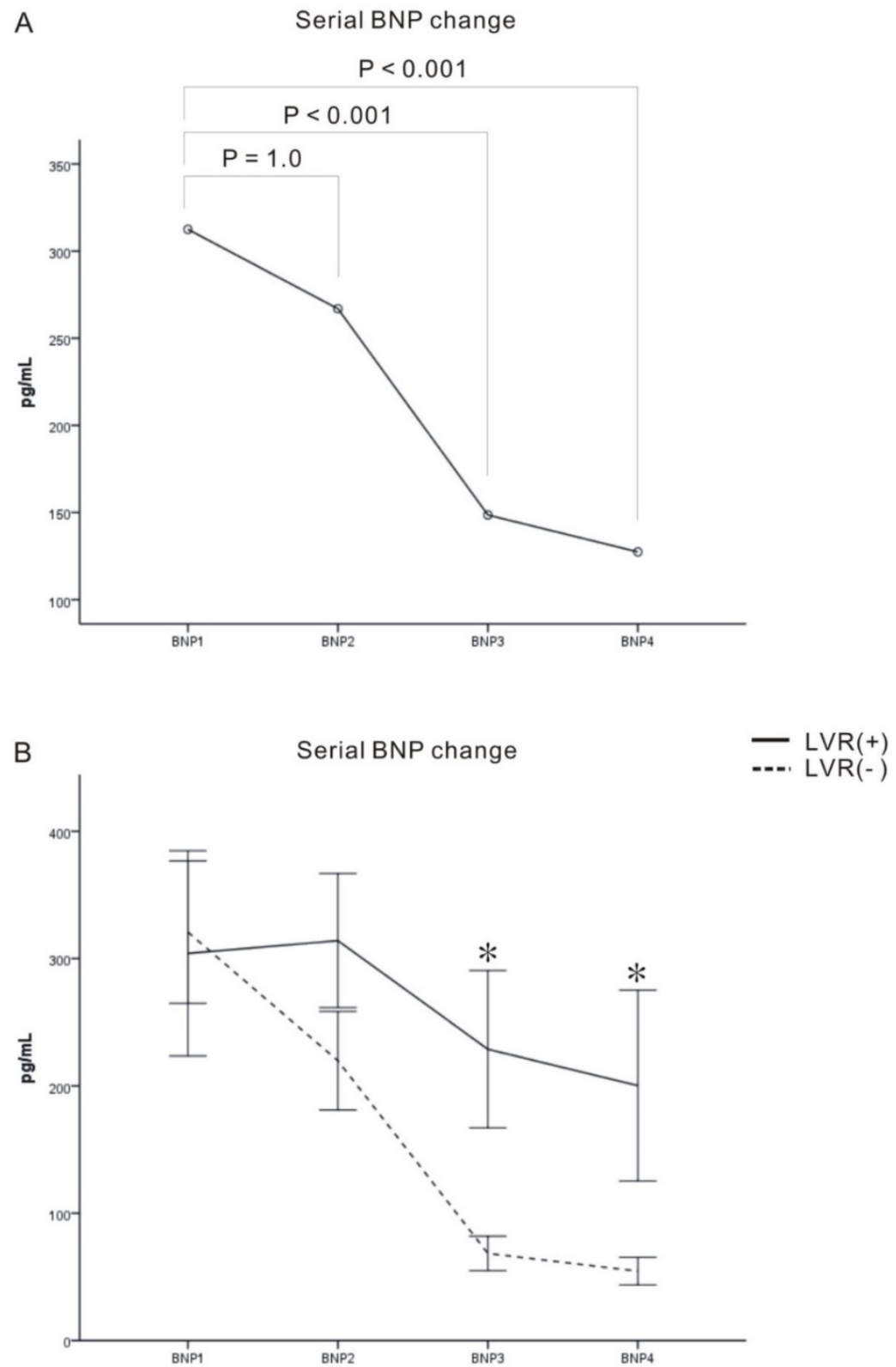

Figure 2. Serial plasma B-type natriuretic peptide (BNP) change in the LV remodeling [LVR(+)] and non LV remodeling [LVR(-)] groups. (A) Pair comparisons showed significant difference between BNPI vs. BNP3 and BNP4, respectively. (B) The BNP level of the two groups showed significant between-subject differences on day 90 and day 180 . The serial BNPs were presented as mean \pm standard error. ${ }^{p} \mathrm{p}<0.05$ between remodeling and no-remodeling group. 


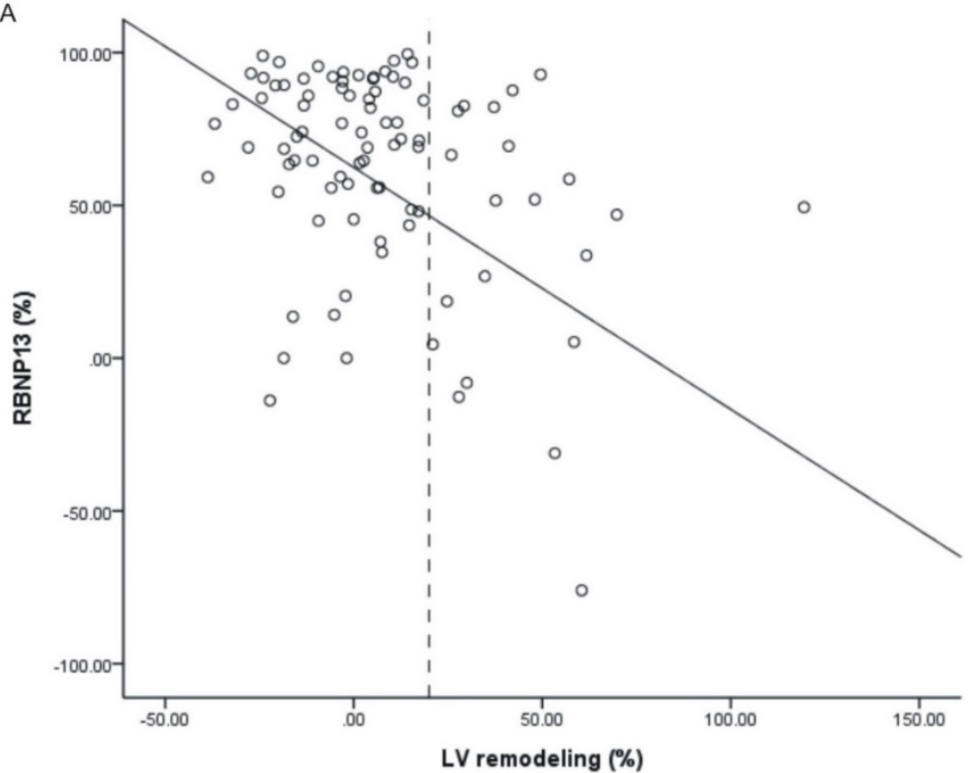

B

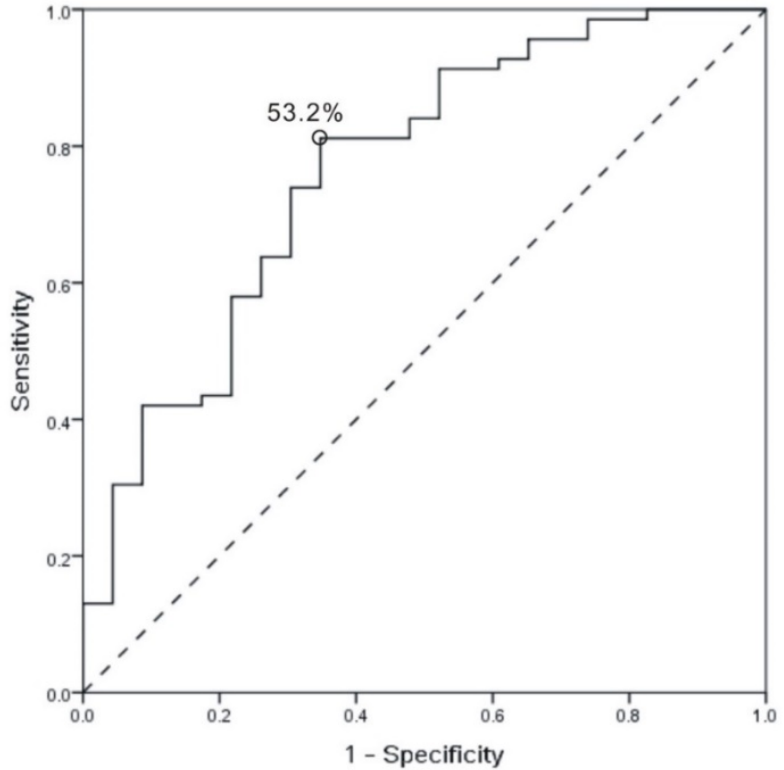

Figure 3. (A) Relationship between 6-month left ventricular (LV) dilatation ratio [LV remodeling (\%)] and 3-month plasma B-type natriuretic peptide (BNP) decrease ratio $\left[R B N P_{13}(\%)\right]$ in patients with $A M I\left(P<0.001, R^{2}=0.219\right.$, decrease in $R B N P_{13}=57.64-1.01 \times L V$ remodeling). The intersection of $L V$ dilatation ratio at $20 \%$ defines a cut-point for LV remodeling. (B) Receiver operating characteristics curve to predict development of half-year LV remodeling by $R B N P_{13}(C u t-o f f$ value of $\left.\operatorname{RBNP}_{13}=53.2 \%, \mathrm{AUC}=0.764, \mathrm{P}<0.001\right)$. AUC: area under the curve.

LVEDVs and LVEFs were calculated on the same days as that samples were taken for BNP measurements. Repeated-measures ANOVA revealed significant within-subject effects for EDV (EDV1 vs. EDV2, EDV1 vs. EDV3, and EDV1 vs. EDV4, all $\mathrm{P}<0.001$ ) (Fig. 4A). The serial change in EDV significantly interacted with $\mathrm{LV}$ remodeling (Fig. 4B, $\mathrm{P}<0.001$ ), though we found no significant between-subject effect verification (Fig. 4B, $\mathrm{P}=0.786$ ). The LVR $(+)$ group had a lower initial EDV and a higher half-year EDV than LVR(-) group $(\mathrm{P}<0.001$ and $\mathrm{P}=0.001$, respectively).

Serial LVEFs comparisons revealed a significant within-subject difference between EF1 and EF3 (Fig 5A: $P=0.008)$ and a significant between-subject difference when comparing the $\operatorname{LVR}(+)$ and $\operatorname{LVR}(-)$ groups (Fig 5B, P = 0.007). The LVR (-) group had a better $\mathrm{EF}$ than $\operatorname{LVR}(+)$ group over the half year follow-up period (EF2: $\mathrm{P}=0.017, \mathrm{EF} 3: \mathrm{P}=0.013, \mathrm{EF} 4 \mathrm{P}$ $=0.002$, respectively). There was no significant interaction between serial LVEF change and LV remodeling. 
A

Serial EDV change
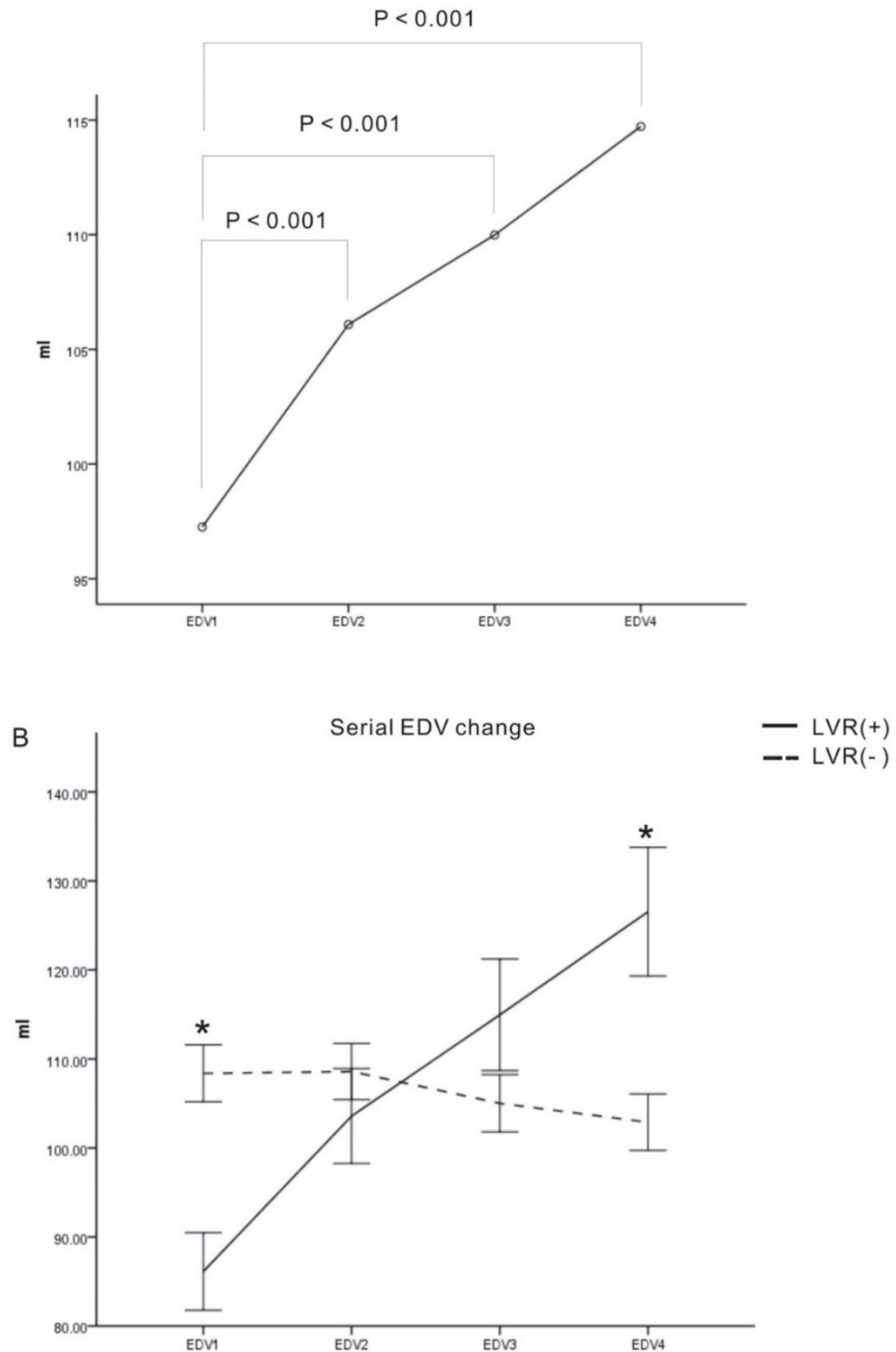

Figure 4. Serial changes in end diastolic volume (EDV). (A) Serial levels of EDV on day-2 (EDV1), day-7 (EDV2), day-90 (EDV3), and day-180 (EDV4) (unit: ml). Pair comparisons showed significant difference between EDV1 vs. EDV2, EDV3 and EDV4, respectively. (B) The LVR(+) group had a lower initial EDV (EDV1) and a higher half-year EDV (EDV4) than the LVR(-) group. The serial EDVs were presented as mean \pm standard error. ${ }^{*} p<0.05$ between remodeling and no-remodeling group.

\section{Early parameter analysis during the first week}

In an attempt to identify an early prognostic marker the first week after AMI, we performed multivariate analysis of age, D-to-B time, S-to-B time, target vessel stent type and peak CK-MB. Only the peak CK-MB time was found to be an independent predictor for half year $L V$ remodeling $(P=0.015)$. The ROC method was used to determine the appropriate peak CK-MB cut-off point, which was $48.2 \mathrm{ng} / \mathrm{ml}$
(Fig. 6A: AUC $=0.672, \mathrm{P}=0.014$ ). No other parameters were found to have a significant influence in our group comparisons.

In our logistic regression analysis, we combine $R_{B N P}$ and peak CK-MB and calculated risk of LV remodeling risk as follows: $\operatorname{Exp}(-0.155)+0.006 \times$ peak $\mathrm{CKMB}-0.03 \times$ RBNP13. This risk equation provided excellent discrimination for half-year LV remodeling and the appropriate cut-off value was 0.285 (Fig. 6A: AUC $=0.818, \mathrm{P}<0.001$ ). Those patients 
with calculated combined result more than 0.285 had low risk for developing half-year LV remodeling, and patients with calculated result less than 0.285 had high risk of half-year LV remodeling. The positive predictive value was $57.1 \%$ and negative predictive value was $88.3 \%$. The initial peak CK-MB level had incremental predicting power (Fig. $6 \mathrm{~B}: \mathrm{P}=0.008$ ).

\section{Discussion}

This study discovered that the $R_{B N P_{13}}$ derived from serial BNP measurements can be used as an independent predictor for half year LV remodeling, supporting the use of serial BNP measurements for dynamic risk stratification for all AMI patients. It also found that peak CK-MB had incremental power to these predictions and thus should be routinely monitored in AMI patients.

Progressive ventricular dilation after acute myocardial infarction can be a main determinant of a patient's prognosis. While previous studies have suggested that early plasma BNP levels can predict short- and long-term prognosis of patients with AMI. [2-6], most of those studies have focused on the anterior wall MI, [3,4] or studied this relationship in STEMI patients only. $[2,5,6]$

A

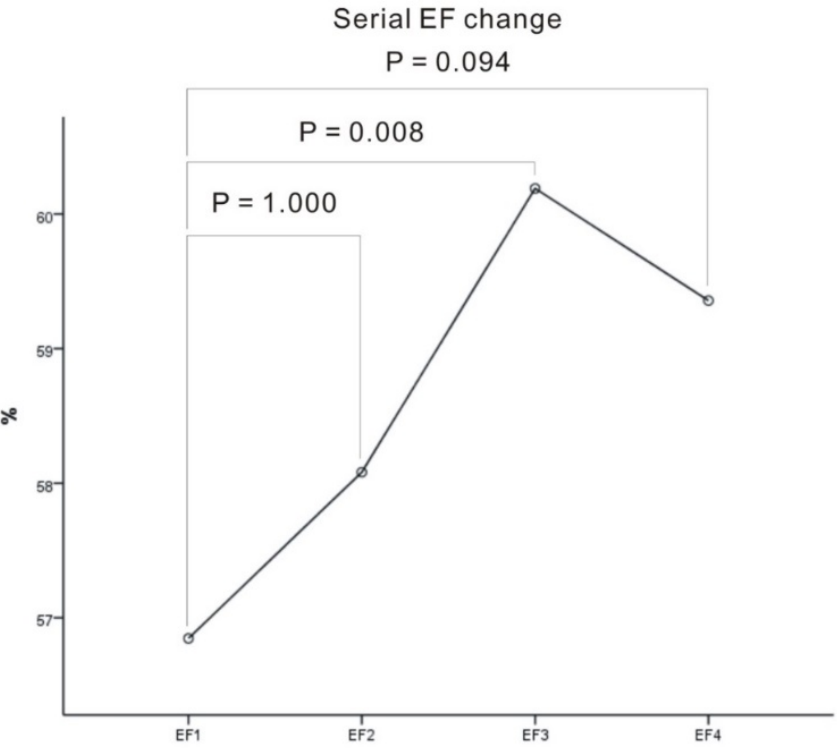

B

Serial EF change

$-\operatorname{LVR}(+)$
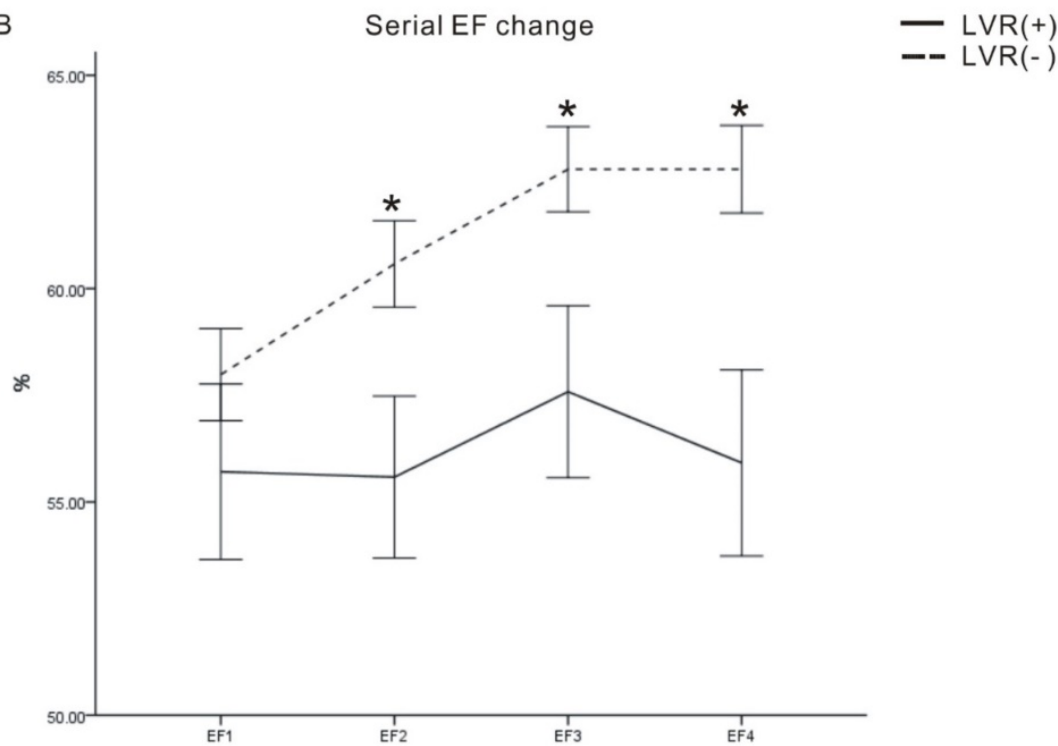

Figure 5. Serial changes in left ventricle ejection fraction (EF). (A) Serial levels of EF on day-2 (EF1), day-7 (EF2), day-90 (EF3), and day-180 (EF4) (unit: \%). Pair comparisons showed significant difference among EF1 and EF3. (B) There were significant between-subject differences in EF2, EF3 and EF4. The serial EFs were presented as mean \pm standard error. ${ }^{*} p<0.05$ between remodeling and no-remodeling group. 
A

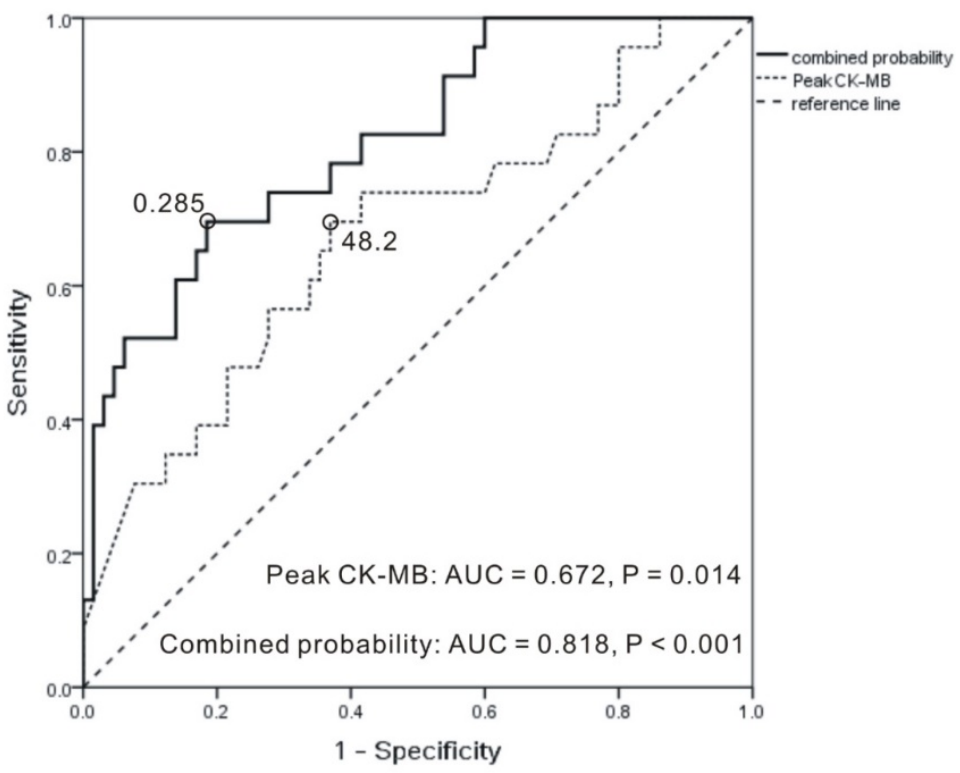

B

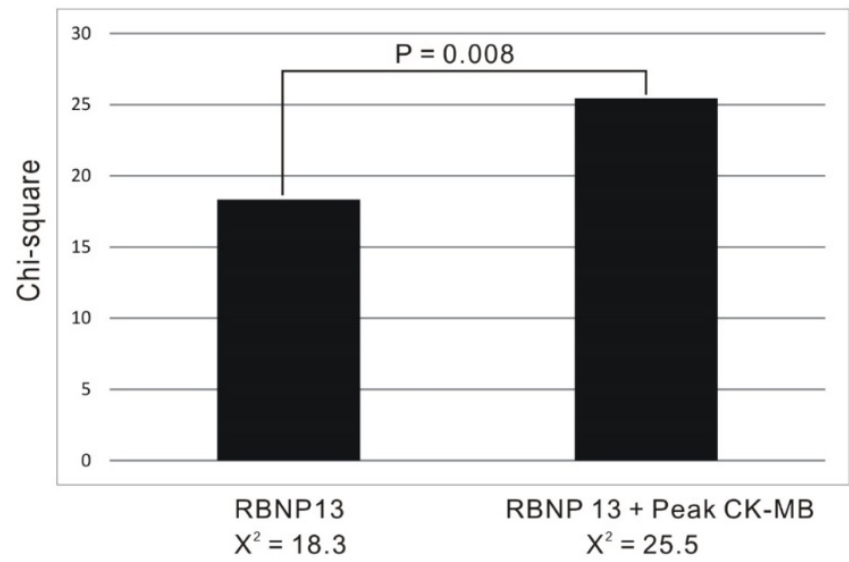

Figure 6. (A). Receiver operating characteristics curve to predict development of half-year LV remodeling by peak CK-MB: Cut-off value of peak CK-MB $=48.2$ $(A \cup C=0.672, P=0.014)$ and combined probability derived from peak $C K-M B$ and 3-month plasma B-type natriuretic peptide decrease ratio $\left(R B N P_{13}\right)(A U C=0.818$, $P<0.001)$. (B). Peak CK-MB offers additional information over $R B N P_{13}$. AUC: area under the curve.

Previous studies have also found that natriuretic peptide values provide information related to outcomes. [7-9] One study suggested that an additional BNP level drawn at 7 weeks post-acute coronary syndrome improved risk stratification. [7] In that study, patients with persistently elevated BNP levels ( $>80 \mathrm{pg} / \mathrm{ml}$ ) had a 4 -fold increased risk for developing cardiovascular events at 10 months compared to those with persistently low BNP levels.

This study found $R_{B N P_{13}}$ to have good differential power in predicting half year remodeling even in our small subgroup populations. If the enrolled patients were further subdivided into STEMI and NSTEMI subgroups, the $R_{B N P} P_{13}$ was found to be a significant predictor of $\mathrm{LV}$ remodeling in both the STEMI subgroup $(\mathrm{P}=0.015)$ and the NSTEMI subgroup $(\mathrm{P}=0.003)$.

Studies using cardiac magnetic resonance imaging have reported peak CK-MB to be an independent predictor for infarct size, LV dysfunction and 1-year clinical outcomes. [10, 11], though one study in which all patients received primary coronary interventions found that peak CK-MB could independently predict LV remodeling. [12] The current study found that the peak CK-MB values could independently but not strongly predict half year LV remodeling. In our subgroup analysis, peak CK-MB was found to acceptably discriminate remodeling $\mathrm{LV}$ from non-remodeling $\mathrm{LV}$ in non-STEMI patients $(\mathrm{P}=0.022)$ but not in STEMI patients $(\mathrm{P}=0.142)$. However, this parameter enhanced the predictive power of serial BNP examination incrementally. The ROC curve of combined $R_{B N P_{13}}$ and peak CK-MB has excellent discrimination for half year remodeling.

Reduction in door-to-balloon time has been shown to be positive prognostic indicator. [13] Both animal studies [14] and clinical studies [15-17] suggest 
that shortening the duration of arterial occlusion caused by infarcts can reduce can infarct sizes and lower cardiac mortality. Time from symptom onset to reperfusion has been documented as an independent predictor of LV functional recovery. [17] However, although both door-to-balloon times and symptom-to-balloon times were significantly different between LVR(+) and LVR(-) group, there parameters were not found in our multivariate analysis to have independent significant predictive power for LV remodeling.

\section{Limitations}

Our study has several limitations. Unlike previous studies $[2,5]$, we did not find that early first-week BNP concentrations (BNP1 and BNP2) to be prognostic indicators. This difference may have been due to the fact that all AMI patients of our study (including STEMI and NSTEMI) were analyzed together. In addition, our patient population was small (97 patients including both STEMI and NSTEMI patients) and thus it might not have been able to demonstrate prognostic significance in the early single BNP blood tests and the S-to-B time, both considered important initial parameters $[2,5,16]$. Another limitation is that, although 110 patients were initially enrolled in this study, nine of our patients died before the 6-month echocardiography evaluation and thus we could not determine predictors of mortality. Nighty-four patients had preserved EF (EF $\geq 40 \%$ ) and only 3 patients had impaired EF (EF < $40 \%)$ at baseline measurement. It means that the study did not enrolled patient with severe heart failure and it could only offered the evidence for patients with EF more than $40 \%$. Future studies with longer evaluation times and larger patient populations are, therefore, needed to demonstrate the effects of BNP levels, S-to-B times, and other parameters on mortality due to AMI.

\section{Conclusions}

In this study, after multivariate adjustment of clinical, laboratory, and angiographic variables, $R \mathrm{BNP}_{13}$ remained a significant independent predictor of 6-month LV remodeling (odds ratio $=0.967, \mathrm{P}=$ 0.003 ). The appropriate cut-off value of $R_{B N P_{13}}$ was $53.2 \%(\mathrm{AUC}=0.764, \mathrm{P}<0.001)$. If the $R_{B N P_{13}}$ was less than $53.2 \%$, the risk of half year LV remodeling increased. In contrast, the risk of half year remodeling decreased if the $\boldsymbol{R B N P}_{13}$ was more than $53.2 \%$. It has high negative predictive value of $87.5 \%$ and moderate positive predictive value of $53.6 \%$. The early peak CK-MB offered an incremental predictive power to serial BNP examination. By combing these two parameters, The ROC curve offered a good discrimination for half-year remodeling (AUC $=0.818, \mathrm{P}<0.001$ ).

\section{Acknowledgments}

This study was supported by grant CMPG6A0141-43 from Chang Gung Memorial Hospital.

\section{Competing Interests}

The authors have declared that no competing interest exists.

\section{References}

1. Sutton MGSJ, Sharpe N. Left Ventricular Remodeling After Myocardial Infarction : Pathophysiology and Therapy. Circulation. 2000;101(25):2981-8. doi: 10.1161/01.cir.101.25.2981.

2. Nagaya N, Nishikimi T, Goto Y, Miyao Y, Kobayashi Y, Morii I, et al. Plasma brain natriuretic peptide is a biochemical marker for the prediction of progressive ventricular remodeling after acute myocardial infarction. American heart journal. 1998;135(1):21-8.

3. White M, Rouleau JL, Hall C, Arnold M, Harel F, Sirois P, et al. Changes in vasoconstrictive hormones, natriuretic peptides, and left ventricular remodeling soon after anterior myocardial infarction. American heart journal. 2001;142(6):1056-64. doi: 10.1067/mhj.2001.119612. PubMed PMID: 11717612.

4. Shuichi T, Satoru S, Takeshi B, Hiroshi T, Naohiko A, Yoshio Y, et al. Predictors of left ventricular remodeling in patients with acute myocardial infarction participating in cardiac rehabilitation. Circulation journal: official journal of the Japanese Circulation Society. 2004;68(3):214-9.

5. Garcia-Alvarez A, Sitges M, Delgado V, Ortiz J, Vidal B, Poyatos S, et al. Relation of plasma brain natriuretic peptide levels on admission for ST-elevation myocardial infarction to left ventricular end-diastolic volume six months later measured by both echocardiography and cardiac magnetic resonance. The American journal of cardiology. 2009;104(7):878-82. doi: 10.1016/j.amjcard.2009.05.025. PubMed PMID: 19766750.

6. Dorobantu M, Fruntelata A-G, Scafa-Udriste A, Tautu O-F. B-type natriuretic peptide (BNP) and left ventricular (LV) function in patients with ST-segment elevation myocardial infarction (STEMI). Mædica. 2010;5(4):243.

7. Ang DS, Kong CF, Kao MP, Struthers AD. Serial bedside B-type natriuretic peptide strongly predicts prognosis in acute coronary syndrome independent of echocardiographic abnormalities. American heart journal. 2009;158(1):133-40. doi: 10.1016/j.ahj.2009.04.024. PubMed PMID: 19540403.

8. Kubánek M, Goode KM, Lánská V, Clark AL, Cleland JG. The prognostic value of repeated measurement of N-terminal pro-B-type natriuretic peptide in patients with chronic heart failure due to left ventricular systolic dysfunction. European journal of heart failure. 2009;11(4):367-77.

9. Januzzi JL, Rehman SU, Mohammed AA, Bhardwaj A, Barajas L, Barajas J, et al. Use of amino-terminal pro-B-type natriuretic peptide to guide outpatient therapy of patients with chronic left ventricular systolic dysfunction. Journal of the American College of Cardiology. 2011;58(18):1881-9.

10. Dohi T, Maehara A, Brener SJ, Généreux P, Gershlick AH, Mehran R, et al. Utility of peak creatine kinase-MB measurements in predicting myocardial infarct size, left ventricular dysfunction, and outcome after first anterior wall acute myocardial infarction (from the INFUSE-AMI trial). The American journal of cardiology. 2015;115(5):563-70.

11. Mayr A, Mair J, Klug G, Schocke M, Pedarnig K, Trieb T, et al. Cardiac troponin $\mathrm{T}$ and creatine kinase predict mid-term infarct size and left ventricular function after acute myocardial infarction: a cardiac MR study. Journal of Magnetic Resonance Imaging. 2011;33(4):847-54.

12. Petronio AS, De Carlo M, Ciabatti N, Amoroso G, Limbruno U, Palagi C, et al. Left ventricular remodeling after primary coronary angioplasty in patients treated with abciximab or intracoronary adenosine. American heart journal. 2005;150(5):1015.1-. e9.

13. Cannon CP, Gibson CM, Lambrew CT, Shoultz DA, Levy D, French WJ, et al. Relationship of symptom-onset-to-balloon time and door-to-balloon time with mortality in patients undergoing angioplasty for acute myocardial infarction. JAMA: the journal of the American Medical Association. 2000;283(22):2941-7.

14. Reimer KA, Lowe J, Rasmussen M, Jennings R. The wavefront phenomenon of ischemic cell death. 1. Myocardial infarct size vs duration of coronary occlusion in dogs. Circulation. 1977;56(5):786-94.

15. Sheiban I, Fragasso G, Rosano GM, Dharmadhikari A, Tzifos V, Pagnotta P, et al. Time course and determinants of left ventricular function recovery after primary angioplasty in patients with acute myocardial infarction. Journal of the American College of Cardiology. 2001;38(2):464-71.

16. De Luca G, Suryapranata H, Zijlstra F, van't Hof AWJ, Hoorntje JCA, Gosselink ATM, et al. Symptom-onset-to-balloon time and mortality in patients with acute myocardial infarction treated by primary angioplasty. 
Journal of the American College of Cardiology. 2003;42(6):991-7. doi: 10.1016/s0735-1097(03)00919-7.

17. Parodi G, Memisha G, Carrabba N, Signorini U, Migliorini A, Cerisano G, et al .

Prevalence, predictors, time course, and long-term clinical implications of left ventricular functional recovery after mechanical reperfusion for acute myocardial infarction. The American journal of cardiology. 2007;100(12):1718-22. doi: 10.1016/j.amjcard.2007.07.022. PubMed PMID: 18082514 . 